\title{
On generalized Nörlund methods of summability
}

\section{Minoru Tanaka}

The object of this paper is to establish some relations between two generalized Nörlund methods and also between two absolute generalized Nörlund methods. Our theorems obtained here generalize many known results, including McFadden's Theorems which state the inclusion relations between two absolute Nörlund methods, and results of I kuko Kayashima.

\section{Introduction}

Let $p=\left\{p_{n}\right\}$ and $\alpha=\left\{\alpha_{n}\right\}$ be given sequences of real numbers such that

$$
(p * \alpha)_{n}=\sum_{\rho=0}^{n} p_{n-\rho} \alpha_{\rho} \neq 0 \quad(n \geq 0)
$$

Given a series $\sum_{n=0}^{\infty} a_{n}$ with its partial sum $s_{n}$, if

$$
t_{n}^{p, \alpha}=\frac{1}{(p \star \alpha)} \sum_{\rho=0}^{n} p_{n-\rho}^{\alpha} \rho_{\rho} \rightarrow s \text { as } n \rightarrow \infty
$$

the series $\sum_{n=0}^{\infty} a_{n}$ is said to be summable $(i, p, \alpha)$ to $s$ and we write

Received 3 October 1978. The author takes this opportunity of expressing his heartfelt thanks to Professors Hiroshi Hirokawa and Ikuko Kayashima for their kind encouragement and valuable suggestions during the preparation of this paper. 
$\sum_{n=0}^{\infty} a_{n}=s(N, p, \alpha)$ (see Borwein [1]). If $\sum_{n=0}^{\infty}\left|t_{n}^{p, \alpha}-t_{n+1}^{p, \alpha}\right|<\infty$, the series $\sum_{n=0}^{\infty} a_{n}$ is said to be summable $|\mathrm{N}, p, \alpha|$ and we write $\sum_{n=0}^{\infty} a_{n} \in|\mathrm{N}, p, \alpha|$. The method $(\mathrm{N}, p, \alpha)$ reduces to the Nörlund method (N,p) when $\alpha_{n}=1$; to the method $(\overline{\mathrm{N}}, \alpha)$ when $p_{n}=1$. Let $\mathrm{A}$ and $B$ be two summability methods. If every series summable (A) to a finite sum is also summable (B) to the same sum, we write $A \subseteq B$. We shall say that $B$ is totally stronger than A (written' B t.s. A ') if, in addition, $\sum_{n=0}^{\infty} a_{n}= \pm \infty$ (A) implies $\sum_{n=0}^{\infty} a_{n}= \pm \infty$ (B). If every series summable $|A|$ is also summable $|B|$, we write $|A| \subseteq|B|$. We shall say that a method $A$ is absolutely regular if every absolutely convergent series is summable $|A|$.

The purpose of this paper is to investigate relations between the methods $(N, p, \alpha)$ and $(N, q, \beta)$, and to establish some conditions for $|N, p, \alpha| \subseteq|N, q, B|$. Our theorems obtained here generalize many known results. We state all the results in $\$ 2$, and they are proved in $\$ \S 4-6$. In $\$ 3$ we state some preliminary lemmas.

Throughout this paper we use the following notations. For sequences $\left\{p_{n}\right\},\left\{q_{n}\right\},\left\{\alpha_{n}\right\}$, and $\left\{\beta_{n}\right\}$, (1.2) $(c * p)_{n}=1 \quad(n=0),=0 \quad(n \geq 1)$, (1.3) $(k * p)_{n}=q_{n} \quad(n \geq 0)$,

$$
K_{\rho}^{n}=\sum_{v=0}^{n} q_{n-v} c_{v \rightarrow \rho} \beta_{v} / \alpha_{v} \quad(n \geq 0) \text {, when } \alpha_{n} \neq 0 \quad(n \geq 0) .
$$

We shall write $\left\{p_{n}\right\} \in M$, if

$$
p_{n}>0, \frac{p_{n+1}}{p_{n}} \leq \frac{p_{n+2}}{p_{n+1}} \leq 1 \quad(n \geq 0),
$$

and also $\left\{p_{n}\right\} \in M(q)$, if 


$$
p_{n}>0, q_{n}>0, \frac{p_{n+1}}{p_{n}} \leq \frac{q_{n+1}}{q_{n}} \quad(n \geq 0) .
$$

We put $\Delta a_{n}=a_{n}-a_{n+1}, \Delta_{n} a_{n, k}=a_{n k}-a_{n+1, k}, a_{-1}=0$. Capital letters $C$ and $H$ are to denote absolute constants, but are not necessarily the same at each occurrence.

\section{Inclusion theorems}

THEOREM 1. If

$$
\begin{aligned}
& \text { (i) }\left\{p_{n}\right\} \in M, \\
& \text { (ii) }\left\{p_{n}\right\} \in M(q), \\
& \text { (iii) } \alpha_{n}>0, B_{n}>0(n \geq 0), \\
& \text { (iv) } B_{n} / \alpha_{n} \geq B_{n+1} / \alpha_{n+1}(n \geq N) \text {, and } \\
& \text { (v) (N, }, \text {, B) is regular, }
\end{aligned}
$$

then $(\mathrm{N}, q, B)$ t.s. $(\mathrm{N}, p, \alpha)$.

The case $N=0$ in condition (iv) is more precise than Das's Theorem ([4], Theorem 1, Case (A)). Putting $\alpha_{n}=\beta_{n}=1 \quad(n \geq 0), p_{n}=q_{n}=1$ $(n \geq 0)$, and $p_{n}=\beta_{n}=1 \quad(n \geq 0)$ in this theorem, we may obtain theorems of Rhoades [13], [14], Lorch [10], and Kuttner and Rhoades [9], respectively.

THEOREM 2. If

$$
\begin{aligned}
& \text { (i) }\left\{p_{n}\right\} \in M, \\
& \text { (ii) }\left\{q_{n}\right\} \in M(p), \\
& \text { (iii) } a_{n}>0(n \geq 0), \\
& \text { (iv) } p_{n} \leq C q_{n}(n \geq 0) \text {, and } \\
& \text { (v) (N, } q, \alpha) \text { is regular, }
\end{aligned}
$$

then $(\mathrm{N}, p, \alpha) \subseteq(\mathrm{N}, q, \alpha)$.

The case in which $a_{n}=1 \quad(n \geq 0)$ is due to Borwein and Cass ([2], 
Theorem 2). But their theorem is more precise than this.

THEOREM 3. If

$$
\begin{aligned}
& \text { (i) }\left\{p_{n}\right\} \in M, \\
& \text { (ii) }\left\{q_{n}\right\} \in M(p), \\
& \text { (iii) } \alpha_{n}>0, B_{n}>0 \quad(n \geq 0) \text {; }
\end{aligned}
$$

and either

$$
\begin{aligned}
& \text { (iv) } \beta_{n} / \alpha_{n} \geq \beta_{n+1} / \alpha_{n+1}(n \geq 0), \\
& \text { (v) } p_{n} \leq C q_{n},(1 * \beta)_{n} \leq H(q * \beta)_{n}(n \geq 0), \\
& \text { (vi) }(\mathrm{N}, q, B) \text { is reguiar, }
\end{aligned}
$$

or

$$
\begin{aligned}
& \text { (iv) } \beta_{n} / \alpha_{n} \leq \beta_{n+1} / \alpha_{n+1} \quad(n \geq 0), \\
& \text { (v) } \beta_{n}(p * \alpha)_{n} \leq C \alpha_{n}(q * \beta)_{n} \quad(n \geq 0), \\
& \text { (vi) }(p * \alpha)_{n} \rightarrow \infty \text { as } n \rightarrow \infty,
\end{aligned}
$$

then $(\mathrm{N}, p, \alpha) \subseteq(\mathrm{N}, q, \beta)$.

The case in which $p_{n}=q_{n}=1 \quad(n \geq 0)$ is known as Riesz's Theorem (see Hardy [6], Theorem 14 with $n_{0}=0$ ).

In the following Theorems $4-7$, we shall suppose that $\alpha_{n} \neq 0$ $(n \geq 0)$.

THEOREM 4. A necessary and sufficient condition that $|\mathrm{N}, p, \alpha| \subseteq|\mathrm{N}, q, \mathrm{~B}|$ is

$$
\sum_{n=k}^{\infty}\left|\sum_{\rho=k}^{n}(p * \alpha)\left\{\frac{K_{\rho}^{n}}{(q * \beta)_{n}}-\frac{K_{p}^{n-1}}{(q * \beta)_{n-1}}\right\}\right| \leq c \quad(k \geq 1) .
$$

The case in which $\alpha_{n}=\beta_{n}=1(n \geq 0)$ is Theorem (2.11) in McFadden [11].

THEOREM 5. If the method ( $\mathrm{N}, q, \beta)$ is absolutely regular, then a necessary and sufficient condition that $|\mathrm{N}, p, \alpha| \subseteq|\mathrm{N}, q, \beta|$ is 
(2.2) $\sum_{n=k}^{\infty}\left|\Delta_{n}\left\{\sum_{\mu=k}^{n-1} \frac{\beta_{\mu}}{\alpha_{\mu}} q_{n-1-\mu} \sum_{\rho=0}^{k-1}(p * \alpha)_{\rho} \frac{c_{\mu-\rho}}{(q * \beta)_{n-1}}\right\}\right| \leq c \quad(k \geq 1)$.

The case in which $p_{n}=q_{n}=1 \quad(n \geq 0)$ is due to Dikshit [5].

THEOREM 6. If

$$
\sum_{\rho=0}^{n}\left|\frac{(p * \alpha)}{(q * \beta)_{n}} k_{\rho}^{n}\right| \leq c \quad(n \geq 0),
$$

(2.4) $\sum_{\rho=k}^{n}(p * \alpha)_{\rho}\left\{k_{\rho}^{n} /(q * \beta)_{n}-k_{\rho}^{n-1} /(q * \beta)_{n-1}\right\} \geq 0$

$$
(k=0,1,2, \ldots, n),
$$

then $|\mathrm{N}, p, \alpha| \subseteq|\mathrm{N}, q, \beta|$.

The case in which $\alpha_{n}=\beta_{n}=1 \quad(n \geq 0)$ is Theorem (2.12) in McFadden [11].

THEOREM 7. If

$$
\begin{aligned}
& (p \star \alpha)_{n}>0,(q * \beta)_{n}>0 \quad(n \geq 0), \\
& \sum_{\rho=0}^{n}(p \star \alpha)_{\rho}\left|K_{\rho}^{n}\right| \leq C(q * \beta)_{n}(n \geq 0),
\end{aligned}
$$

and either

$$
K_{\rho}^{n} /(q * B)_{n}-K_{\rho}^{n+1} /(q * B)_{n+1} \geq 0 \quad(n-\rho \geq N)
$$

or

$$
k_{\rho}^{n} /(q * \beta)_{n}-k_{\rho}^{n+1} /(q * \beta)_{n+1} \leq 0 \quad(n-\rho \geq N)
$$

then $|\mathrm{N}, p, \alpha| \subseteq|\mathrm{N}, q, B|$.

When $\alpha_{n}=\beta_{n}=1 \quad(n \geq 0)$ in this theorem, the cases (2.7) and (2.8) are due to McFadden [11] and Kayashima [8], respectively.

In the following Theorems 8 to 11 , we shall suppose that $p_{n}>0$, $q_{n}>0, \alpha_{n}>0$, and $\beta_{n}>0(n \geq 0)$.

THEOREM 8. If 


$$
\begin{gathered}
(q * \beta)_{n} \leq(q * \beta)_{n+1} \quad(n \geq 0), \\
\beta_{n} \leq C \alpha_{n}, \quad(p * \alpha)_{n} \leq H(q * \beta)_{n}(n \geq 0),
\end{gathered}
$$

and either

$$
k_{\rho}^{n} \geq 0, \quad K_{\rho}^{n} \geq K_{\rho}^{n+1} \quad(n-\rho \geq N)
$$

or

$$
K_{\rho}^{n} \leq 0, \quad K_{\rho}^{n} \leq K_{\rho}^{n+1} \quad(n-\rho \geq N),
$$

then $|\mathrm{N}, p, \alpha| \subseteq|\mathrm{N}, q, \beta|$.

When $\alpha_{n}=\beta_{n}=1 \quad(n \geq 0)$ in this theorem, the cases (2.11) and

(2.12) are also due to McFadden [11] and Kayashima [8], respectively.

From these results we get the following theorems.

THEOREM 9. If

$$
\begin{aligned}
& \text { (i) }\left\{p_{n}\right\} \in M, \\
& \text { (ii) } \Delta q_{n}>0(n \geq 0), \\
& \text { (iii) } q_{0} / p_{0}=\beta_{0} / \alpha_{0}, \\
& \text { (iv) }(q * \beta)_{n} \leq(q * \beta)_{n+1}(n \geq 0) \text {, }
\end{aligned}
$$

and either

A: $\quad$ (v) $B_{n} / \alpha_{n} \geq \beta_{n+1} / \alpha_{n+1} \quad(n \geq 0)$,

$$
\begin{aligned}
& \text { (vi) } \Delta q_{n} / \Delta p_{n} \leq \Delta q_{n+1} / \Delta p_{n+1} \quad(n \geq 0), \\
& \text { (vii) } \beta_{n} / \alpha_{n} \geq \Delta q_{n} / \Delta p_{n}(n \geq 0),
\end{aligned}
$$

or

B: $\quad$ (v) $\beta_{n} / \alpha_{n} \leq \beta_{n+1} / \alpha_{n+1} \quad(n \geq 0)$,

(vi) $\Delta q_{n} / \Delta p_{n} \geq \Delta q_{n+1} / \Delta p_{n+1} \quad(n \geq 0)$,

(vii) $\beta_{n} / \alpha_{n} \leq \Delta q_{n} / \Delta p_{n}(n \geq 0)$,

(viii) $(p * \alpha)_{n} \leq C(q * B)_{n}(n \geq 0)$, 
then $|\mathrm{N}, p, \alpha| \subseteq|\mathrm{N}, q, \beta|$.

The case in which $\alpha_{n}=\beta_{n}=1(n \geq 0)$ is due to Kayashima [8].

THEOREM 10. If

(i) $\left\{p_{n}\right\} \in M$, and

(ii) $\beta_{n} / \alpha_{n} \geq \beta_{n+1} / \alpha_{n+1} \quad(n \geq 0)$,

then $|\mathrm{N}, p, \alpha| \subseteq|\overline{\mathrm{N}}, \mathrm{B}|$.

The cases in which $\alpha_{n}=\beta_{n}(n \geq 0)$ and $\alpha_{n}=1 \quad(n \geq 0)$ are due to Das [3] and Kayashima [7], respectively.

THEOREM 11.' If either

A: $\quad$ (i) $q_{n} \leq q_{n+1}, q_{n+1} / q_{n} \geq q_{n+2} / q_{n+1}(n \geq 0)$,

(ii) $\beta_{n} / \alpha_{n} \geq \beta_{n+1} / \alpha_{n+1}(n \geq 0)$,

or

B: (i) $q_{n} \geq q_{n+1}, \quad(q * \beta)_{n} \leq(q * B)_{n+1}(n \geq 0)$,

(ii) $B_{n+1}(1 * \alpha)_{n} \leq C \alpha_{n+1}(q * \beta)_{n+1}(n \geq 0)$,

then $|\bar{N}, \alpha| \subseteq|N, q, B|$.

The cases $B_{n}=1(n \geq 0)$ in Condition $A, B_{n}=1 \quad(n \geq 0)$ in Condition B, and $q_{n}=1(n \geq 0)$ in Condition B are due to Kayashima [7], Dikshit [5], and Sunouchi [15], respectively.

\section{Preliminary lemmas}

LEMMA 1. Necessary and sufficient conditions for the method $(N, p, a)$ to be regular are:

(i) $\sum_{\rho=0}^{n}\left|p_{n-\rho} \alpha_{\rho}\right|=O\left((p * \alpha){ }_{n}\right)$ as $n+\infty$,

(ii) $p_{n-\rho} \alpha_{\rho}=o\left((p * \alpha)_{n}\right)$ as $n \rightarrow \infty$, for each $\rho \geq 0$.

This follows from Toeplitz's Theorem (see Hardy [6], Theorem 2). If 
$\left\{p_{n}\right\}$ and $\left\{\alpha_{n}\right\}$ are positive sequences, then condition $(i)$ above is satisfied.

LEMMA 2. Let $\alpha_{n} \neq 0 \quad(n \geq 0)$. Then necessary and sufficient conditions that $(N, p, \alpha) \subseteq(N, q, B)$ are

$$
\begin{aligned}
\sum_{\rho=0}^{n}(p * \alpha)_{\rho} K_{\rho}^{n} & =o\left((q * \beta)_{n}\right), \\
K_{\rho}^{n} & =o\left((p * \beta)_{n}\right) \text { as } n+\infty \text {, for each } \rho \geq 0 .
\end{aligned}
$$

This is due to Das ([4], Lemma I).

LEMMA 3. Let $p_{n}>0, q_{n}>0, \alpha_{n}>0$, and $\beta_{n}>0$ for alz $n \geq 0$. Then necessary and sufficient conditions that $(\mathrm{N}, q, \beta)$ t.s. (N, $p, \alpha)$ are (3.2) and

$$
K_{\rho}^{n} \geq 0 \quad(n \geq \rho \geq N)
$$

Proof. After Das ([4], Lemma 1), given $t_{n}^{p, \alpha}$ and $t_{n}^{q, \beta}$ which are defined by (1.1), we get

$$
t_{n}^{q, \beta}=\sum_{\rho=0}^{n} a_{n \rho} t_{\rho}^{p, \alpha},
$$

where

$$
a_{n \rho}= \begin{cases}\left.\frac{(p \star \alpha)}{(q \star \beta)}\right)_{n} k_{\rho}^{n} & (n \geq \rho), \\ 0 & (n<\rho) .\end{cases}
$$

If $s_{n}=1 \quad(n \geq 0)$ in $(1.1)$, then $t_{n}^{p, \alpha}=1$, and also $t_{n}^{q, \beta}=1$. Hence

$$
\sum_{\rho=0}^{n} a_{n \rho}=1 \quad(n \geq 0) .
$$

Since the transformation defined by (3.4) is positive under our conditions, it is sufficient for the proof to show that this transformation is regular (see Hardy [6], Theorem 10). Hence, by Lemma 1, we need only show that (3.1) is satisfied. Now by (3.3) and (3.5) we have 


$$
\sum_{\rho=0}^{n}\left|(p * \alpha) \rho K_{\rho}^{n}\right| \leq 2 \sum_{\rho=0}^{N-1}(p * \alpha)_{\rho}\left|K_{\rho}^{n}\right|+(q * \beta)_{n},
$$

and by $(3.2)$,

$$
\sum_{\rho=0}^{N-1}(p * \alpha)_{\rho}\left|K_{\rho}^{n}\right|=O\left((q * B){ }_{n}\right)
$$

Hence we get

$$
\sum_{\rho=0}^{n}\left|(p \star \alpha){ }_{\rho} K_{\rho}^{n}\right|=O\left((p \star \beta){ }_{n}\right),
$$

which is $(3.1)$.

Conversely the necessity of conditions (3.2) and (3.3) is immediately obtained from Hurwitz's Theorem (Hardy [6], Theorem 10) and from Lemma 2. Thus the proof is complete.

LEMMA 4. If $\left\{p_{n}\right\} \in M$, then

$$
c_{0}>0, c_{n} \leq 0 \quad(n \geq 1), \sum_{n=0}^{\infty} c_{n} \geq 0 .
$$

This lerma is due to Kaluza (see Hardy [6], Theorem 22).

LEMMA 5. If $\left\{p_{n}\right\} \in M$, then

$$
k_{n} \geq 0 \quad(n \geq 0) \text { whenever }\left\{p_{n}\right\} \in M(q) \text {, }
$$

or

$$
k_{0}>0, k_{n} \leq 0 \quad(n \geq 1) \text { whenever }\left\{q_{n}\right\} \in M(p) .
$$

Cases (3.7) and (3.8) are due to Hardy ([6], p. 69) and Borwein and Cass ([2], p. 102), respectively.

LEMMA 6. Let $y_{n}=\sum_{\rho=0}^{\infty} a_{n \rho \rho} x_{\rho}(n \geq 0)$. Then necessary and sufficient conditions that $\sum_{n=0}^{\infty}\left|\Delta y_{n}\right|<\infty$ whenever $\sum_{n=0}^{\infty}\left|\Delta x_{n}\right|<\infty$ are

$$
\sum_{\rho=0}^{\infty} a_{n \rho} \text { converges for all } n \geq 0 \text {, }
$$




$$
\sum_{n=0}^{\infty}\left|\sum_{\rho=k}^{\infty}\left(a_{n \rho}-a_{n-1, \rho}\right)\right| \leq C \quad(k \geq 0) .
$$

This is due to Mears [12] and Sunouchi [15], independently.

\section{LEMMA 7. If}

$$
\begin{aligned}
& \text { (i) } p_{n}>0, \alpha_{n}>0 \quad(n \geq 0) \text {, } \\
& \text { (ii) }(p * \alpha)_{n} \leq(p * \alpha)_{n+1}(n \geq 0) \text {, and } \\
& \text { (iii) } p_{n} \geq p_{n+1}(n \geq N) \text {, }
\end{aligned}
$$

then the method $(\mathrm{N}, p, \alpha)$ is absolutely regular.

Proof. We show that the conditions of Lemma 6 are satisfied with

$$
\begin{aligned}
a_{n \rho} & =p_{n-\rho}^{\alpha} \rho /(p * \alpha)_{n} & & (n \geq \rho) \\
& =0 & & (n<\rho) .
\end{aligned}
$$

Then (3.9) holds. Hence it suffices to prove that

$$
\sum_{n=k}^{\infty}\left|\sum_{\rho=k}^{n}\left\{\frac{p_{n-\rho} \rho_{\rho}}{(p * \alpha)_{n}}-\frac{p_{n-1-\rho^{\alpha} \rho}}{(p * \alpha)_{n-1}}\right\}\right| \leq c \quad(k \geq 1) .
$$

Now by using our conditions we have

$$
\begin{aligned}
I_{k}^{n} & =\sum_{\rho=k}^{n}\left\{\frac{p_{n-\rho} \rho_{\rho}}{(p * \alpha)_{n}}-\frac{p_{n-1-\rho}^{\alpha} \rho}{(p * \alpha)_{n-1}}\right\} \\
& =\sum_{\rho=0}^{k-1} \alpha_{\rho}\left\{\frac{p_{n-1-\rho}}{(p * \alpha)_{n-1}}-\frac{p_{n-\rho}}{(p * \alpha)_{n}}\right\} \\
& \geq 0 \quad(n \geq N+k, k \geq 1) .
\end{aligned}
$$

Hence

$$
\begin{aligned}
\sum_{n=k}^{\infty}\left|I_{k}^{n}\right| & =\sum_{n=k}^{N+k-1}\left|I_{k}^{n}\right|+\sum_{n=N+k}^{\infty} I_{k}^{n} \\
& \leq 2 N+1 \quad(k \geq 1) .
\end{aligned}
$$

This completes the proof of Lemma 7 .

The case in which $\alpha_{n}=1 \quad(n \geq 0)$ is due to Mears [12]. 


\section{Proof of Theorems 1-3}

4.1. Proof of Theorem 1. For the proof it is sufficient to show that conditions (3.2) and (3.3) are satisfied. Now by Abel's transformation, we have

$$
k_{\rho}^{n}=\sum_{\nu=\rho}^{n-1} \Delta\left(\frac{\beta_{\nu}}{\alpha_{v}}\right) \sum_{\mu=\rho}^{\nu} q_{n-\mu} c_{\mu-\rho}+\frac{B_{n}}{\alpha_{n}} k_{n-\rho} .
$$

Then using (3.6), and by condition (iv),

$$
\sum_{\nu=\rho}^{n-1} \Delta\left(\frac{\beta_{\nu}}{\alpha_{\nu}}\right) \sum_{\mu=\rho}^{\nu} q_{n-\mu^{c}}{ }_{\mu-\rho} \geq 0 \quad(\rho \geq N),
$$

and also, by (3.7), $k_{n-\rho} \geq 0(n \geq \rho)$. Hence, from (4.1.1), we get condition (3.3). Next, also by (iv) and using (3.6),

$$
k_{\rho}^{n} \leq c_{0} \frac{\beta_{\rho}}{\alpha_{\rho}} q_{n-\rho} \quad(\rho \geq N) .
$$

On the other hand, for $0 \leq \rho \leq N-1$,

$$
\left|K_{\rho}^{n}\right| \leq c_{0} q_{n-\rho}\left\{\sum_{v=\rho}^{N-1}\left|\Delta\left(\frac{\beta_{v}}{\alpha_{v}}\right)\right|+\frac{\beta_{N}}{\alpha_{N}}\right\} .
$$

Therefore, by use of Lemma 1 (ii), we obtain condition (3.2).

This completes the proof.

4.2. Proof of Theorem 2. We show that the conditions of Lemma 2 of the case $\alpha_{n}=B_{n}(n \geq 0)$ are satisfied. Now by $(i),(i i)$, and (iv), using $(3.8)$,

$$
\begin{aligned}
\sum_{\rho=0}^{n} p_{\rho}\left|k_{n-\rho}\right| & =2 k_{\rho} p_{n}-q_{n} \\
& =o\left(q_{n}\right) .
\end{aligned}
$$

Hence, by $(i v)$, we get

$$
\begin{aligned}
\sum_{\rho=0}^{n}\left|(p * \alpha)_{\rho} k_{n-\rho}\right| & =\sum_{\nu=0}^{n} \alpha_{v}\left(\sum_{\rho=v}^{n} p_{\rho-\nu}\left|k_{n-\rho}\right|\right) \\
& =o\left((q * \alpha)_{n}\right),
\end{aligned}
$$


which is (3.1), because $k_{\rho}^{n}=k_{n-\rho}(n \geq \rho)$ when $\alpha_{n}=\beta_{n} \quad(n \geq 0)$. Next, from (4.2.1) for fixed $\rho \geq 0$,

$$
k_{n-\rho}=o\left(q_{n-\rho}\right) \text {. }
$$

Therefore, by use of Lemma 1 (ii), we can obtain condition (3.2), and thus the proof is complete.

4.3. Proof of Theorem 3. Case I. Let conditions A hold. By Abel's transformation, we have

$$
K_{\rho}^{n}=\sum_{\nu=\rho}^{n-1} \Delta_{\nu}\left(q_{n-\nu} \sum_{\mu=\rho}^{\nu} c_{\mu-\rho} \frac{\beta_{\mu}}{\alpha_{\mu}}\right)+q_{0} \sum_{\mu=\rho}^{n} c_{\mu-\rho} \frac{\beta_{\mu}}{\alpha_{\mu}} .
$$

Then, under our conditions and by use of (3.6),

$$
\begin{aligned}
\sum_{\nu=\rho}^{n-1} \Delta_{\nu} q_{n-\nu}\left(\sum_{\mu=\rho}^{\nu} c_{\mu-\rho} \frac{\beta_{\mu}}{\alpha_{\mu}}\right) & \leq 0 \quad(n>\rho), \\
q_{0} \sum_{\mu=\rho}^{n} c_{\mu-\rho} \frac{\beta_{\mu}}{\alpha_{\mu}} & \geq 0 \quad(n>\rho) .
\end{aligned}
$$

Hence, by (3.5), we have

$$
\text { (4.3.1) } \sum_{\rho=0}^{n}(p * \alpha)_{\rho}\left|K_{\rho}^{n}\right|=2 q_{0} \sum_{\rho=0}^{n}(p * \alpha)_{\rho} \sum_{\mu=\rho}^{n} c_{\mu-\rho} \frac{\beta_{\mu}}{\alpha_{\mu}}-(q * \beta)_{n} \text {. }
$$

Now, by (1.2),

$$
(p * \alpha * c)_{n}=\alpha_{n}(n \geq 0) \text {. }
$$

Hence, by $A(v)$, we get

$$
\begin{aligned}
\sum_{\rho=0}^{n}(p * \alpha)_{\rho} \sum_{\mu=\rho}^{n} c_{\mu-\rho} \frac{\beta_{\mu}}{\alpha_{\mu}} & =\sum_{\mu=0}^{n} \frac{\beta}{\alpha_{\mu}} \sum_{\rho=0}^{\mu}(p * \alpha)_{\rho} c_{\mu-\rho} \\
& =\sum_{\mu=0}^{n} \frac{\beta_{\mu}}{\alpha_{\mu}}(p * \alpha * c) \\
& =(1 * \beta)_{n} \\
& \leq H(q * \beta)_{n} .
\end{aligned}
$$

Therefore, from (4.3.1), we obtain condition (3.3). Next, by use of (3.6), we have 
Methods of summability

393

$$
K_{\rho}^{n} \leq c_{0} q_{n-\rho} \frac{\beta_{\rho}}{\alpha_{\rho}} .
$$

On the other hand, by A (iv),

$$
k_{\rho}^{n} \leq k_{n-\rho} \frac{\beta_{\rho}}{\alpha_{\rho}} .
$$

Since conditions (i), (ii), and (iv) of Theorem 2 are fulfilled, (4.2.1) holds. Hence we can obtain, by A ( $i v)$ and by use of Lemma 1 (ii), condition (3.2). Thus the desired conclusion of Case I follows from Lemma 2.

CASE II. Let conditions B hold. Using (3.6) and (3.8), we have, by $B(i v)$,

$$
k_{\rho}^{n} \leq \frac{{ }_{\rho}^{\beta}}{\alpha_{\rho}} k_{n-\rho} \leq 0 \quad(n \geq \rho) .
$$

Thus

$$
\begin{aligned}
\sum_{\rho=0}^{n}(p * \alpha)_{\rho}\left|K_{\rho}^{n}\right| & =2(p * \alpha)_{n} q_{0} c_{0} \frac{\beta_{n}}{\alpha_{n}}-\sum_{\rho=0}^{n}(p * \alpha)_{\rho} K_{\rho}^{n} \\
& =2 q_{0} c_{0} \frac{\beta_{n}(p * \alpha)_{n}}{\alpha_{n}(q * \beta)_{n}}(q * \beta)_{n}-(q * \beta)_{n} .
\end{aligned}
$$

Hence, by B $(v)$, we obtain condition (3.1). Next, by Abel's transformation, we have, for $n>\rho$,

$$
\begin{aligned}
K_{\rho}^{n} & =\sum_{\nu=\rho}^{n-1} \Delta_{\nu}\left(a_{n-\nu} \frac{\beta_{\nu}}{\alpha_{\nu}}\right)\left(\sum_{\mu=\rho}^{\nu} c_{\mu-\rho}\right)+\frac{B_{n}}{\alpha_{n}} q_{0}\left(\sum_{\mu=\rho}^{n} c_{\mu-\rho}\right) \\
& =g(n, \rho)+h(n, \rho) \text {, say. }
\end{aligned}
$$

Then by $\mathrm{B}(i v)$, and since $\left\{q_{n}\right\}$ is non-increasing,

$$
\begin{aligned}
\Delta_{v}\left(q_{n-v} \frac{\beta_{v}}{\alpha_{v}}\right) & =q_{n-v} \frac{\beta_{\nu}}{\alpha_{v}}-q_{n-1-\nu} \frac{\beta_{v+1}}{\alpha_{v+1}} \\
& \leq 0 \quad(n>v) .
\end{aligned}
$$

Using (3.6), we see that

$$
g(n, \rho)=\sum_{\nu=\rho}^{n}\left(q_{n-\nu} \frac{\beta_{\nu}}{\alpha_{\nu}}\right)\left(\sum_{\mu=\rho}^{\nu} c_{\mu-\rho}\right) \leq 0,
$$

https://doi.org/10.1017/S0004972700008935 Published online by Cambridge University Press 


$$
h(n, \rho)=\frac{\beta_{n}}{\alpha_{n}} q_{0}\left(\sum_{\mu=\rho}^{n} c_{\mu-\rho}\right) \geq 0 .
$$

Hence we get, from (4.3.3),

$$
g(n, \rho) \leq K_{\rho}^{n} \leq 0 \quad(n>\rho)
$$

On the other hand

$$
\begin{aligned}
g(n, \rho) & \geq \sum_{\nu=\rho}^{n-1} \Delta_{v}\left(q_{n-v} \frac{\beta_{\nu}}{\alpha_{v}}\right) c_{0} \\
& \geq-c_{0} q_{0} \frac{\beta_{n}}{\alpha_{n}},
\end{aligned}
$$

and so we obtain, by $B(v)$ and $B(v i)$, for fixed $\rho \geq 0$,

$$
\frac{g(n, p)}{(q \star \beta)_{n}} \geq\left(-c_{0} q_{0}\right) \frac{\beta_{n}(p \star \alpha)_{n}}{\alpha_{n}(q \star \beta)_{n}} \frac{1}{(p \star \alpha)_{n}} \rightarrow 0 \text { as } n \rightarrow \infty \text {. }
$$

Therefore we can get condition (3.2). Thus the desired conclusion of Case II also follows.

Thus the proof of Theorem 3 is complete.

\section{Proof of Theorems 4-8}

5.1. Proof of Theorem 4: Let $a_{n \rho}$ be given by (3.4). Then by (3.5) we have (3.9). Condition (2.1) is the same as (3.10). Hence we can get the required result from Lemma 6 .

5.2. Proof of Theorem 5. We show that condition (2.1) is satisfied. Now by $(4.3 .2)$,

$$
\begin{aligned}
\sum_{\rho=k}^{n}(p & * \alpha)_{\rho} K_{\rho}^{n} \\
& =\left(\sum_{\rho=0}^{n}-\sum_{\rho=0}^{k-1}\right\} \\
& =(p * \beta)_{n}-\left\{\sum_{\mu=0}^{k-1} \frac{\beta_{\mu}}{\alpha_{\mu}} q_{n-\mu}(p * \alpha * c)_{\mu}+\sum_{\mu=n}^{n} \frac{\beta}{\alpha_{\mu}} q_{n-\mu} \sum_{\rho=0}^{k-1}(p * \alpha)_{\rho} c_{\mu-\rho}\right\} \\
& =(q * \beta)_{n}-\left\{\sum_{\mu=0}^{k-1} \beta_{\mu} q_{n-\mu}+\sum_{\mu=n}^{n} \frac{\beta_{\mu}}{\alpha_{\mu}} q_{n-\mu} \sum_{\rho=0}^{k-1}(p * \alpha)_{\rho} c_{\mu-\rho}\right\} .
\end{aligned}
$$


Thus we have

$$
\begin{aligned}
\left.\sum_{\rho=k}^{n}(p * \alpha)\left\{\frac{k_{\rho}^{n}}{\rho(p * \beta)_{n}}-\frac{k_{\rho}^{n-1}}{(p * \beta)_{n-1}}\right\}=\left\{\Delta\left(\Delta_{n} \sum_{\mu=0}^{k-1} \beta_{\mu} q_{n-1-\mu}\right) /\left((q * \beta)_{n-1}\right)\right)\right\} \\
+\left\{\Delta_{n}\left(\sum_{\mu=k}^{n-1} \frac{\beta_{\mu}}{\alpha_{\mu}} q_{n-1-\mu} \sum_{\rho=0}^{k-1}(p * \alpha)_{\rho}{ }^{c} \mu-\rho /(q * \beta)_{n-1}\right)\right\} .
\end{aligned}
$$

Now a condition of the absolute regularity of the method $(N, q, B)$ is equivalent to

$$
\sum_{n=k}^{\infty}\left|\Delta_{n}\left(\left(\sum_{\mu=0}^{k-1} \beta_{\mu} q_{n-1-\mu}\right) /\left((q * \beta)_{n-1}\right)\right)\right| \leq C \quad(k \geq 1) .
$$

Hence condition (2.1) is equivalent to (2.2). Thus the required conclusion follows from Lemma 4.

5.3. Proof of Theorem 6. Let $a_{n \rho}$ be given by (3.4), and $k \geq 1$. Then under our conditions we have, for $m>k$,

$$
\begin{aligned}
\sum_{n=k}^{m}\left|\sum_{\rho=k}^{n}\left(a_{n \rho}-a_{n-1, \rho}\right)\right| & =\sum_{\rho=k}^{m} \sum_{n=\rho}^{m}\left(a_{n \rho}-a_{n-1, \rho}\right) \\
& \leq \sum_{\rho=0}^{m}\left|a_{m \rho}\right| \leq c .
\end{aligned}
$$

Hence we get (2.1) when $m+\infty$, and thus the proof is complete.

5.4. Proof of Theorem 7. Let $a_{n \rho}$ be given by (3.4), and $k \geq 1$. Now, for $m \geq+k$,

$$
\begin{aligned}
\sum_{n=k}^{m}\left|\sum_{\rho=k}^{n}\left(a_{n \rho}-a_{n-1, \rho}\right)\right| & =\left(\sum_{n=k}^{N+k-1}-\sum_{n=N+k}^{m}\right) \\
& =\Sigma_{1}+\Sigma_{2} \text {, say. }
\end{aligned}
$$

Then, by (2.6), we have

$$
\begin{aligned}
\Sigma_{1} & =\sum_{n=k}^{N+k-1}\left|\sum_{\rho=k}^{n}\left(a_{n \rho}-a_{n-1, \rho}\right)\right| \\
& \leq 2 H C .
\end{aligned}
$$

CASE I. Let (2.5), (2.6), and (2.7) hold. When $n \geq N+k$ and $\rho=0,1, \ldots, k-1$, it is clear that $n-\rho-1>N$, and so by (2.5) and 
from (2.6) we get

$$
\begin{aligned}
\sum_{\rho=k}^{n}\left(a_{n \rho}-a_{n-1, \rho}\right) & =\sum_{\rho=0}^{k-1}(p * \alpha)\left\{\frac{k_{\rho}^{n-1}}{(g * \beta)_{n-1}}-\frac{k_{\rho}^{n}}{(q * \beta)_{n}}\right\} \\
& \geq 0 .
\end{aligned}
$$

Hènce, by (2.6),

$$
\begin{aligned}
\Sigma_{2} & =\sum_{n=N+k}^{m} \sum_{\rho=k}^{n}\left(a_{n \rho}-a_{n-1, \rho}\right) \\
& \leq \sum_{\rho=0}^{m}\left|a_{m \rho}\right|+\sum_{\rho=0}^{N+k-1}\left|a_{+k-1, \rho}\right| \\
& \leq 2 C .
\end{aligned}
$$

Therefore, applying $(5.4 .2)$ and $(5.4 .3)$ to $(5.4 .1)$, we can obtain (2.1). CASE II. Let (2.5), (2.6), and (2.8) hold. Similarly we have, by (2.8) and (2.6),

$$
\begin{aligned}
\Sigma_{2} & =\sum_{n=N-1}^{m}(-1) \sum_{\rho=k}^{n}\left(a_{n \rho}-a_{n-1, \rho}\right) \\
& \leq \sum_{\rho=0}^{N+k-1}\left|a_{+k-1, \rho}\right|+\sum_{\rho=0}^{m}\left|a_{m \rho}\right| \\
& \leq 2 C .
\end{aligned}
$$

Hence we can also obtain (2.1). The statements of Theorem 7 thus follow from Theorem 4.

5.5. Proof of Theorem 8. We shall show that the conditions of Theorem 7 are fulfilled for each case.

CASE I. Let $(2.9),(2.10)$, and (2.11) hold. Condition (2.5) is clear. Let $n \geq N$; then we have, by (2.11),

$$
\text { (5.5.1) } \begin{aligned}
\sum_{\rho=0}^{n} \frac{(p * \alpha)_{\rho}}{(q * \beta)_{n}}\left|K_{\rho}^{n}\right| & =\int_{\rho=0}^{n-N}+\sum_{\rho=n-N+1}^{n}\left|\frac{(p * \alpha)}{(q * \beta)_{n}}\right| K_{\rho}^{n} \mid \\
& \leq 1+2 \max _{n-N+1 \leq \rho \leq n}\left\{\left|K_{\rho}^{n}\right|\right\} \cdot\left\{\sum_{\rho=n-N+1}^{n} \frac{(p * \alpha)_{\rho}}{(q * \beta)_{n}}\right\} .
\end{aligned}
$$

Here, by (2.9) and (2.10), 


$$
\sum_{\rho=n-N+1}^{n} \frac{(p * \alpha)_{\rho}}{(q * \beta)_{n}} \leq \sum_{\rho=n-N+1}^{n} \frac{(p * \alpha)_{\rho}}{(q * \beta)_{\rho}} \leq N H .
$$

On the other hand, when $n-+1 \leq \rho \leq N-1$, then $0 \leq n-\rho \leq N-1$, and so, by (2.10),

$$
\begin{aligned}
\left|K_{\rho}^{n}\right| & \leq \sum_{\mu=\rho}^{n} \frac{\beta_{\mu}}{\alpha_{\mu}} q_{n-\mu}\left|c_{\mu-\rho}\right| \\
& \leq c\left(\sum_{i=0}^{N-1} q_{i}\right)\left(\sum_{j=0}^{N-1}\left|c_{j}\right|\right) .
\end{aligned}
$$

Hence we get

$$
\max _{n-N+1 \leq \rho \leq n}\left\{\left|K_{\rho}^{n}\right|\right\} \leq C<\infty .
$$

Therefore from (5.5.1) we can obtain condition (2.6). Also by (2.9) and (2.10),

$$
\begin{aligned}
\frac{K_{\rho}^{n}}{(q * B)_{n}}-\frac{K_{\rho}^{n+1}}{(q * \beta)_{n+1}} & \geq \frac{1}{(q * B)_{n}}\left(K_{\rho}^{n}-K_{\rho}^{n+1}\right) \\
& \geq 0 \quad(n-\rho \geq N),
\end{aligned}
$$

which is (2.7).

CASE II. Let (2.9), (2.10), and (2.12) hold. The proof of this case follows in a similar manner as above, and so it may be omitted. Finally suppose that $K_{\rho}^{n} \geq 0$ and $K_{\rho}^{n} \geq K_{\rho}^{n+1} \quad(n-\rho \geq 0)$. Then we have

$$
\sum_{\rho=0}^{n} \frac{(p \star \alpha)_{\rho}}{(q \star \beta)_{n}}\left|K_{\rho}^{n}\right|=1 \quad(n \geq 0),
$$

and hence the desired result immediately follows.

Thus our theorem is proved.

\section{Proof of Theorems 9-11}

6.1. Proof of Theorem 9. CASE I. Let conditions A hold. In this case we shall show that the conditions of Theorem 8 with $N=0$ are fulfilled. Then under our hypotheses it suffices to show that $K_{\rho}^{n} \geq 0$ and 
$K_{\rho}^{n} \geq K_{\rho}^{n+1}(n-\rho \geq 0)$. Since $\left\{p_{n}\right\} \in M,(3.6)$ holds, and so by $\mathrm{A}(v)$, we have

$$
k_{\rho}^{n} \geq \frac{\beta}{\alpha_{\rho}} k_{n-\rho} .
$$

Then by $\mathrm{A}(v)$ and $\mathrm{A}(v i i)$ we get $q_{0} / p_{0} \geq \Delta q_{n} / \Delta p_{n}(n \geq 0)$, and thus we can obtain $k_{n} \geq 0 \quad(n \geq 0)$ (see Kayashima [8]). Hence

$$
K_{p}^{n} \geq 0 \quad(n-\rho \geq 0)
$$

On the other hand, by $A(v i)$ and $A$ (vii), and since $c_{n+1-p} \leq 0$ $(n-\rho \geq 0)$,

$$
\begin{aligned}
K_{\rho}^{n}-K_{\rho}^{n+1} & =\sum_{k=0}^{n-\rho} \frac{\beta_{\rho+k}}{\alpha_{\rho+k}} c_{k} \Delta_{n} q_{n-\rho-k}-\frac{\beta_{n+1}}{\alpha_{n+1}} c_{n+1-\rho} q_{0} \\
& \geq \frac{\beta_{\rho}}{\alpha_{\rho}} \Delta_{n} q_{n-\rho} \sum_{k=0}^{n-\rho} c_{k} \frac{\Delta_{n} q_{n-\rho-k}}{\Delta_{n} q_{n-\rho}}-\frac{\beta_{n+1}}{\alpha_{n+1}} c_{n+1-\rho} q_{0} \\
& \geq \frac{\beta_{\rho}}{\alpha_{\rho}} \frac{\Delta_{n} q_{n-\rho}}{\Delta_{n} p_{n-\rho}} \sum_{k=0}^{n-\rho} c_{k} \Delta_{n} p_{n-\rho-k}-\frac{\beta_{n+1}}{\alpha_{n+1}} c_{n+1-\rho} q_{0} \\
& \geq \frac{\beta_{\rho}}{\alpha_{\rho}} \frac{\Delta_{n} q_{n-\rho}}{\Delta_{n} p_{n-\rho}} p_{0} c_{n+1-\rho}-\frac{\beta_{n+1}}{\alpha_{n+1}} c_{n+1-\rho} q_{0} \\
& \geq\left(-c_{n+1-\rho}\right)_{0}\left(\frac{\beta_{n+1}}{\alpha_{n+1}}-\frac{\Delta_{n} q_{n-\rho}}{\Delta_{n} p_{n-\rho}}\right) \\
& \geq 0 \quad(n-\rho \geq 0) .
\end{aligned}
$$

Thus we have the conclusion.

CASE II. Let conditions B hold. Similarly it suffices to show that (2.12) is satisfied with $N=1$. Now by B (vi) and B (vii) we have $q_{0} / p_{0} \leq \Delta q_{n} / \Delta p_{n}(n \geq 0)$, and also we can get $k_{n} \leq 0 \quad(n \geq 1)$ (see Kayashima [8]). Therefore

$$
K_{\rho}^{n} \leq \frac{\beta_{\rho}}{\alpha_{\rho}} k_{n-\rho} \leq 0 \quad(n-\rho \geq 1) .
$$

Next, by $B(v i)$ and $B(v i i)$, we get in a similar manner, 


$$
\begin{aligned}
K_{\rho}^{n}-K_{\rho}^{n+1} & \leq\left(-c_{n+1-\rho}\right) q_{0}\left\{\frac{\beta_{n+1}}{\alpha_{n+1}}-\frac{\Delta_{n} q_{n-\rho}}{\Delta_{n} p_{n-\rho}}\right\} \\
& \leq 0 \quad(n-\rho \geq 1),
\end{aligned}
$$

which is (2.12). This completes the proof of Theorem 9.

6.2. Proof of Theorem 10. It suffices to show that the conditions of Theorem 8 are fulfilled with $N=0$ and $q_{n}=1 \quad(n \geq 0)$. Then (2.9) is obvious. Now by $(i)$ and $(i i)$, and by use of (3.6), we have, for $n-p \geq 0$,

$$
\begin{aligned}
k_{\rho}^{n} & =\sum_{\mu=\rho}^{n} \frac{\beta_{\mu}}{\alpha_{\mu}} c_{\mu-\rho} \geq \frac{\beta_{\rho}}{\alpha_{\rho}}\left(\sum_{i=0}^{n-\rho} c_{i j}\right) \geq 0, \\
k_{\rho}^{n}-k_{\rho}^{n+1} & =\frac{\beta_{n+1}}{\alpha_{n+1}}\left(-c_{n+1-\rho}\right) \geq 0 .
\end{aligned}
$$

Thus the proof is complete.

6.3. Proof of Theorem 11. CASE I. Let conditions A hold. We show that the conditions of Theorem 6 with $p_{n}=1 \quad(n \geq 0)$ are satisfied. Now by (1.3) we get

$$
c_{0}=1, c_{1}=-1, c_{n}=0 \quad(n \geq 2) .
$$

Let $a_{n \rho}$ be given by (3.4); then, by A (i) and A (ii),

$$
a_{n \rho}=\frac{(1 * \alpha)}{(q * \beta)_{n}} \Delta_{\rho}\left(\frac{\beta}{\alpha_{\rho}} q_{n-\rho}\right) \geq 0 \quad(n-\rho \geq 0) .
$$

Thus we have, immediately, condition (2.3). Hence it is sufficient to show that condition (2.3) holds. By Abel's transformation, ve have

$$
\sum_{\rho=k}^{n} a_{n \rho}=\left(\sum_{\rho=0}^{n-k} \beta_{n-\rho} q_{\rho f}\right) /\left((q * B)_{n}\right)+\frac{(1 * \alpha)_{k-1} \beta_{k} q_{n-k}}{\alpha_{k}(q * \beta)_{n}} .
$$

Hence 


$$
\begin{aligned}
I^{n} & =\sum_{\rho=k}^{n}\left\{a_{n \rho}-a_{n-1, \rho}\right\} \\
& =\left\{\left(\sum_{\rho=0}^{n-k} \beta_{n-\rho} q_{\rho}\right) /\left((q * \beta){ }_{n}\right)-\left(\sum_{\rho=0}^{n-1-k} B_{n-1-\rho} q_{\rho}\right) /\left((q * \beta)_{n-1}\right)\right\} \\
& +\frac{\beta_{k}(1 * \alpha)_{k-1}}{\alpha_{k}}\left\{\frac{q_{n-k}}{(q * B)_{n}}-\frac{q_{n-1-k}}{(q * B)}\right\} \\
& =I_{1}^{n}+I_{2}^{n}, \text { say. }
\end{aligned}
$$

since $\left\{q_{n+1} / q_{n}\right\}$ is non-increasing,

$$
\begin{aligned}
\left.\sum_{\rho=0}^{n-k} \beta_{n-\rho^{q}}\right)(q * \beta)_{n-1}-(q * \beta) & \left(\sum_{\rho=0}^{n-1-k} \beta_{n-1-\rho} q_{\rho}\right) \\
& =\beta_{n} q_{0}\left(\sum_{j=0}^{k-1} q_{n-1-j} \beta_{j}\right)+\sum_{i=k}^{n-1} \sum_{j=0}^{k-1} \beta_{k} \beta_{j}\left(q_{n-i} q_{n-1-j}-q_{n-j} q_{n-1-i}\right) \\
& \geq 0 .
\end{aligned}
$$

Hence we obtain $I_{1}^{n} \geq 0$. Thus if $I_{2}^{n} \geq 0$, then it is clear that $I^{n} \geq 0$. On the other hand suppose that $I_{2}^{n}<0$. Then, since $\left\{q_{n}\right\}$ is non-decreasing and $\left\{\beta_{n} / \alpha_{n}\right\}$ is non-increasing, we get, for $0 \leq m \leq k$,

$$
\alpha_{m} \frac{\beta_{k}}{\alpha_{k}}\left\{\frac{q_{n-k}}{(q * \beta)_{n}}-\frac{q_{n-1-k}}{(q * \beta)_{n-1}}\right\} \geq \beta_{m}\left\{\frac{q_{n-m}}{(q * \beta)_{n}}-\frac{q_{n-1-m}}{(q * \beta)_{n-1}}\right\}
$$

and so

$$
\begin{aligned}
I_{2}^{n} & =\frac{(1 * \alpha)_{k-1} \beta_{k}}{\alpha_{k}}\left\{\frac{q_{n-k}}{(q \star \beta)_{n}}-\frac{q_{n-1-k}}{(q * B)_{n-1}}\right\} \\
& \geq \sum_{m=0}^{k-1}\left\{\frac{\beta_{m} q_{n-m}}{(q \star B)_{n}}-\frac{B_{m}{ }_{n-1-m}}{(q * B)_{n-1}}\right\} .
\end{aligned}
$$

Therefore we have

$$
\begin{aligned}
& I^{n} \geq\left\{\left(\sum_{\rho=0}^{n-k} \beta_{n-\rho} q_{\rho}\right) /\left((q * \beta)_{n}\right)-\left(\sum_{\rho=0}^{n-1-k} \beta_{n-1-\rho} q_{\rho}\right) /\left((q * \beta)_{n-1}\right)\right\} \\
& -\sum_{m=0}^{k-1}\left\{\frac{\beta_{m} q_{n-m}}{(q * \beta)_{n}}-\frac{\beta_{m} q_{n-1-m}}{(q * \beta)_{n-1}}\right\}
\end{aligned}
$$

$=0$. 
Thus the required conclusion follows.

- CASE II. Let conditions B hold. By Lemma 6 we see that ( $\mathrm{H}, q, \beta$ ) is absolutely regular. Hence it is sufficient to show that condition (2.2) of Theorem 5 is satisfied with $p_{n}=1 \quad(n \geq 0)$. Now, by use of (6.3.1) and by $B(i)$, we have

$$
\begin{aligned}
& \sum_{n=k}^{\infty}\left|\Delta_{n}\left\{\sum_{\mu=k}^{n-1} \frac{\beta_{\mu}}{\alpha_{\mu}} q_{n-1-\mu} \sum_{\rho=0}^{\mu}(p * \alpha)_{\rho}{ }^{c}{ }_{\mu-\rho} /(p * \beta)_{n-1}\right\}\right| \\
&=\frac{\beta_{k}}{\alpha_{k}}(1 * \alpha)_{k-1} \sum_{n=k+1}^{\infty}\left\{\frac{q_{n-1-k}}{(q * \beta)_{n-1}}-\frac{q_{n-k}}{(q * \beta)_{n}}\right\}+\frac{q_{0}}{(q * \beta)_{n}} \\
& \leq 2 q_{0} \frac{\beta_{k}(1 * \alpha)_{k-1}}{\alpha_{k}(q * \beta)_{k}} .
\end{aligned}
$$

Hence it follows from hypothesis B (ii) that condition (2.2) is satisfied. Thus the proof of Theorem 11 is complete.

\section{References}

[1] D. Borwein, "On products of sequences", J. London Math. Soc. 33 (1958), 352-357.

[2] D. Borwein and F.P. Cass, "Strong Nörlund summability", Math. 2. 103 (1968), 94-111.

[3] G. Das, "On some methods of summability", Quart. J. Math. Oxford Ser. 17 (1966), 244-256.

[4] G. Das, "On some methods of summability (II)", Quart. I. Math. Oxford Ser. 19 (1968), 417-431.

[5] G.D. Dikshit, "On inclusion relation between Riesz and Nörlund means", Indian J. Math. 7 (1965), 73-81.

[6] G.H. Hardy, Divergent series (Clarendon Press, Oxford, 1949).

[7] Ikuko Kayashima, "On relations between Nörlund and Riesz means", Pacific J. Math. 49 (1973), 391-396.

[8] Ikuko Kayashima, "Inclusion theorems for absolute Norlund summability", submitted. 
[9] Brian Kuttner and B.E. Rhoades, "Relations between $\left(N, p_{n}\right)$ and $\left(\bar{N}, p_{n}\right)$ summability", Proc. Edinburgh Math. Soc. (2) 16 (1968), 109-116.

[10] Lee Lorch, "Supplement to a theorem of Cesàro", Scripta Math. 23 (1957), 163-165.

[11] Leonard McFadden, "Absolute Nörlund summability", Duke Math. J. 9 (1942), 168-207.

[12] Florence M. Mears, "Absolute regularity and Nörlund mean", Ann. of Math. (2) 38 (1937), 594-601.

[13] B.E. Rhoades, "On the total inclusion for Nörlund methods of summability", Math. 2. 96 (1967), 183-188.

[14] Billy E. Rhoades, "On the total inclusion for Nörlund methods of summability. II", Math. 2. 113 (1970), 171-172.

[15] Gen-ichirô Sunouchi, "Notes on Fourier analysis (XVIII): absolute summability of series with constant terms", Tôhoku Math. J. (2) 1 (1949), 57-65.

Department of Mathematics,

Faculty of Science,

Chiba University,

Chiba,

Japan. 